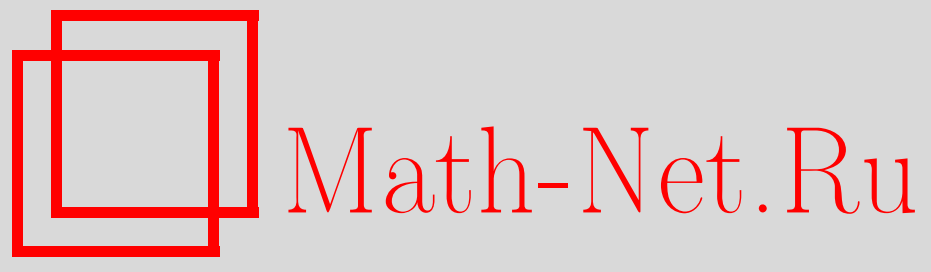

Р. Г. Новиков, И. А. Тайманов, Преобразование Мутара и двумерные многоточечные дельтаобразные потенциалы, УМН, 2013, том 68, выпуск 5, 181-182

DOI: https://doi.org/10.4213/rm9548

Использование Общероссийского математического портала Math-Net.Ru подразумевает, что вы прочитали и согласны с пользовательским соглашением http://www . mathnet.ru/rus/agreement

Параметры загрузки:

IP : 54.147 .182 .235

26 апреля 2023 г., 15:13:01

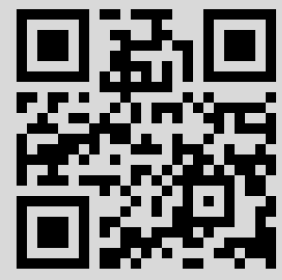




\section{Преобразование Мутара и двумерные многоточечные дельтаобразные потенциалы}

\section{Р. Г. Новиков, И. А. Тайманов}

Пусть $H$ - двумерный оператор Шрёдингера $H=-\Delta+U=-4 \bar{\partial} \partial+U$, где $\partial=$ $(\partial / \partial x-i \partial / \partial y) / 2, x, y \in \mathbb{R}$, и $\omega-$ формальное решение уравнения

$$
H \omega=0 .
$$

Преобразование Мутара паре $H$ и $\omega$ ставит в соответствие оператор

$$
\widetilde{H}=-4 \bar{\partial} \partial+\widetilde{U}=-4 \bar{\partial} \partial+U-8 \bar{\partial} \partial \log \omega
$$

такой, что для любого решения $\varphi$ уравнения $H \varphi=0$ функция $\theta$, удовлетворяющая системе

$$
(\omega \theta)_{z}=-i \omega^{2}\left(\frac{\varphi}{\omega}\right)_{z}, \quad(\omega \theta)_{\bar{z}}=i \omega^{2}\left(\frac{\varphi}{\omega}\right)_{\bar{z}},
$$

удовлетворяет уравнению $\widetilde{H} \theta=0$. Функция $\theta$ определена по модулю $1 / \omega$ в силу константы интегрирования в правой части (3).

Недавно преобразование Мутара, первоначально возникшее в теории поверхностей, было использовано для построения специальных классов двумерных потенциалов и разрушающихся решений уравнения Веселова-Новикова [1], [2].

В отличие от [1], где рассматриваются регулярные потенциалы, настоящая заметка посвящена многоточечным дельтаобразным потенциалам. Мы рассматриваем также собственные функции Фаддеева [3] соответствующих операторов $H$ на нулевом уровне энергии. Эти собственные функции определяются условиями

$$
H \psi=0, \quad \psi(z, \bar{z}, \lambda)=e^{\lambda z}(1+o(1)) \quad \text { при } z \rightarrow \infty, \lambda \in \mathbb{C} \backslash\{0\} .
$$

При этом $\psi=e^{\lambda z}\left(1+a(\lambda, \bar{\lambda}) / z+e^{\bar{\lambda} \bar{z}-\lambda z} b(\lambda, \bar{\lambda}) / \bar{z}+o(1 /|z|)\right), z \rightarrow \infty$, где $a, b-$ обобщенные данные "рассеяния" Фаддеева на нулевом уровне энергии.

Теорема 1. Формальное применение преобразования Мутара к нулевому потенциалу $U=0$ с использованием полиномиальной по z функции $\omega=P(z)=\prod_{k=1}^{N}\left(z-z_{k}\right)$ приводит к многоточечному дельтаобразному потенииалу

$$
\widetilde{U}(z)=-8 \pi \sum_{k=1}^{N} \delta\left(z-z_{k}\right) .
$$

Для этого потенциала собственные функции Фаддеева на нулевом уровне энергии принимают вид

$$
\psi=e^{\lambda z}\left(1+\frac{2}{P} \sum_{k=1}^{N} \frac{(-1)^{k} P^{(k)}(z)}{\lambda^{k}}\right), \quad \text { дде } P^{(k)}(z)=\partial^{k} P(z) .
$$

Кроме того, для этих собственных функиий $a=-2 N / \lambda u b \equiv 0$.

Доказательство этой теоремы основано на решении системы (3) относительно $\psi=\theta$ при $\omega=P(z)$ и $\varphi=i e^{\lambda z}$ и на прямых вычислениях. Однако необходимо уточнить смысл уравнения Шрёдингера с таким потенциалом. Фактически, в этом случае

Работа выполнена во время визита второго автора в Centre de Mathématiques Appliquées (Ecole Polytechnique) в июле 2013 г. Первый автор поддержан ФЦП № 14.A18.21.0866 Министерства образования и науки РФ, второй - программой “Фундаментальные проблемы нелинейной динамики" Президиума РАН и грантом 1431/ГФ МОН Республики Казахстан.

DOI: $10.4213 / \mathrm{rm} 9548$ 
мы рассматриваем систему Мутара (3) с $\omega=P(z)$ как подходящую регуляризацию уравнения Шрёдингера $\widetilde{H} \theta=0$ с потенциалом $\widetilde{U}$ из теоремы 1 . При этом для $N=1$ уравнение Шрёдингера $(-\Delta+\widetilde{U}) \psi=0$ с $\widetilde{U}$ и $\psi$ из теоремы 1 формально выполнено при следующих соглашениях: $\bar{\partial}\left(e^{\lambda z}(1 / z)^{2}\right)=e^{\lambda z}(2 / z) \bar{\partial}(1 / z)=2 \pi e^{\lambda z} \delta(z) / z$.

Отметим, что функции $\psi$ из (4) существенно отличаются от собственных функций Фаддеева, найденных в [4], [5], для операторов Шрёдингера с многоточечными дельтаобразными потенциалами. Причина состоит в том, что в [4], [5] оператор с таким потенциалом заменен на его регуляризацию, восходящую к [6], тогда как в настоящей заметке мы формально работаем с исходными потенциалами, рассматривая регуляризацию уравнения $\widetilde{H} \theta=0$, задаваемую системой Мутара (3).

При этом, ввиду свойства $b \equiv 0$ для $\psi$ из (4), потенциалы из теоремы 1 могут рассматриваться как "безотражательные" в смысле обобщенных данных "рассеяния" Фаддеева $a, b$. В этом случае функции $\psi$ из (4) аналогичны собственным функциям Фаддеева, найденным в [7] для некоторых регулярных потенциалов.

В [1], [8] преобразование Мутара было расширено до преобразования решений уравнения Веселова-Новикова [9]

$$
U_{t}=\partial^{3} U+\bar{\partial}^{3} U+3 \partial(U V)+3 \bar{\partial}(U \bar{V})=0, \quad-4 \bar{\partial} V=\partial U .
$$

Это уравнение имеет форму Манакова $H_{t}=H A+B H$, где $A$ и $B$ - дифференциальные операторы. Если $U$ удовлетворяет (5) и $\omega$ удовлетворяет (1) и уравнению

$$
\left(\partial_{t}+A\right) \omega=0,
$$

то расширенное преобразование Мутара от $U$ имеет тот же вид (2) и дает новое решение уравнения (5). Для нулевого потенциала $U=V=0$ мы имеем $A=\partial^{3}+\bar{\partial}^{3}$, и $\omega(z, t)=P(z, t)=\prod_{k=1}^{N}\left(z-z_{k}(t)\right)$ удовлетворяет (6) тогда и только тогда, когда $\partial P / \partial t=\partial^{3} P / \partial z^{3}$. Последнее уравнение описывает алгебраическую динамику нулей $P(z, t)$ (такая динамика по другой причине была рассмотрена в [1]). Формальное применение расширенного преобразования Мутара приводит к потенциалу $\widetilde{U}(z, t)=$ $-8 \pi \sum_{k=1}^{N} \delta\left(z-z_{k}(t)\right)$, который, по-видимому, может рассматриваться как формальное решение уравнения (5).

\section{Список литературы}

[1] И. А. Тайманов, С. П. Царев, ТMФ, 157:2 (2008), 188-207. [2] И. А. Тайманов, С. П. Царев, Докл. АН ССCP, 420:6 (2008), 744-745. [3] Л. Д. Фаддеев, Докл. АН CCCP, 165:3 (1965), 514-517. [4] P. G. Grinevich, R. G. Novikov, Phys. Lett. A, 376:12-13 (2012), 1102-1106; arXiv: 1110.3157. [5] P. G. Grinevich, R. G. Novikov, arXiv: 1211.0292. [6] Ф. А. Березин, Л. Д. Фаддеев, Докл. АН СССР, 137 (1961), 1011-1014. [7] И. А. Тайманов, С. П. Царев, ТМФ, 176:3 (2013), 408-416, arXiv: 1208.4556. [8] H.-C. Hu, S.-Y. Lou, Q.-P. Liu, Chinese Phys. Lett., 20:9 (2003), 1413-1415. [9] А. П. Веселов, С. П. Новиков, Докл. АН СССР, 279:1 (1984), 20-24.

P. Г. Новиков (R. G. Novikov)

CNRS, Centre de Mathématiques Appliquées, Ecole Polytechnique, Palaiseau, France;

Московский физико-технический институт

E-mail: novikov@cmap.polytechnique.fr

\section{И. А. Тайманов (I. A. Taimanov)}

Институт математики им. С. Л. Соболева СО РАН

E-mail: taimanov@math.nsc.ru
Представлено В. М. Бухштабером Принято редколлегией 03.08.2013 